\title{
Mais où vas-tu, qualité?
}

\section{Philippe Luchsinger}

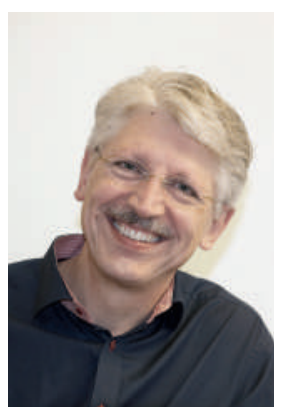

Philippe Luchsinger
Correspondance: Dr Philippe Luchsinger Praxis im Rosenfeld Betpurstrasse 32 8910 Affoltern am Albis philippe.luchsinger[at] hin.ch
Le sang de certains collègues ne fait qu'un tour à la simple idée de débats portant sur la qualité: qu'attendent-ils de nous? Que va-t-il advenir? Notre travail est qualifié de remarquable, nos patients sont satisfaits: que demande-t-on de plus?

Dès les premiers jours, MFE s'est fixé comme objectif d'accorder à la notion de qualité la place nécessaire. Le point essentiel de ces réflexions était: la qualité doit être utile à nos membres et, ainsi, à nos patients. L'association des Médecins de famille et de l'enfance Suisse a également reçu les consignes de manière contractuelle: après sa création, MFE s'est vu confier par la SSMG, la SSMI, la SSP et le CMPR la tâche de se saisir de la question de la qualité. L’accent a été mis sur quatre points: le cercle de qualité, les patients, les collaborateurs et la relève. La démarche sur la qualité ne doit pas être utopiste et irréaliste, mais simple et proche des fondamentaux; elle doit être comprise par chaque membre de MFE et recueillir son approbation. Des étapes simples, menées dans un environnement familier, doivent conduire à ce que tous les médecins de famille et de l'enfance puissent participer activement au questionnement sur la qualité, et non pas à ce qu'ils se sentent écrasés sous son poids. Un objectif très ambitieux!

Aujourd'hui, la situation a changé: la nouvelle Société de Médecine Interne Générale, intégrée au plan, est disposée à se saisir de la question de la qualité et a déjà effectué les premières étapes, avant même sa création officielle. Un premier concept a été élaboré au sein d'un groupe de travail composé de représentants de la SSMI, de la SSMG et de MFE, et a déjà fait l'objet d'une com- munication officielle (PrimaryCare. 2015;15(8);134). La voie est ainsi libre pour ancrer ici aussi des sujets pour lesquels il est clair qu'un débat s'impose au sein de la société de discipline médicale. Le domaine des recommandations, en particulier, est ici, à n'en pas douter, à la bonne place. C'est précisément ce thème, avec les thèmes qui en découlent tels que le shared decision making, qui nécessitera de très nombreuses ressources. La commission Qualité de MFE a-t-elle désormais fait son temps? Toutes les tâches vont-elles être reprises par cette nouvelle commission intégrée à la société de discipline médicale? Certainement pas! Les différentes dimensions de la qualité en médecine présentent des

\section{La commission Qualité de MFE reste indispen-} sable étant donné que la SSMIG ne peut pas assumer tous les rôles.

aspects politiques essentiels qui ne peuvent pas être assumés par la SSMIG, telle que s'appellera cette société de discipline médicale. Lorsqu'il s'agit de donner de la visibilité au travail sur la qualité et de montrer aux politiques et à la population à quel point ils sont bien pris en charge, le poids politique de MFE est indispensable. Ce poids politique est également nécessaire au sein du corps médical, ne serait-ce que pour continuer à intégrer la pédiatrie, pour être présent dans les discussions avec les assureurs à propos du travail fourni par nos membres, et pour avoir notre mot à dire en matière de tarifs et d'indemnités. Le travail sur la qualité a sa valeur, la SSMIG l'élabore et MFE le défend, avec la force et les conséquences habituelles. 